\section{Treatment of acquired immune insulin resistance with i. v. immunoglobulins}

Dear Sir,

Type I diabetes treated with insulin is associated with the production of anti-insulin antibodies that possibly trap insulin in immune complexes, change its pharmacokinetics, and worsen glycaemic control. Intraperitoneal (i.p.) insulin infusion has been shown to stimulate the production of anti-insulin antibodies in roughly $50 \%$ of the patients [1] but clinical consequences occur in only a few patients. We report about a 65year-old woman who had Type I diabetes for 11 years and was treated by i.p. insulin infusion with an implantable pump. She had two catheter encapsulations requiring surgical treatment after 18 and 27 months. After the second treatment, she developped a severe insulin resistance, requiring insulin up to 450 IU/day whatever the route of injection (i.p., i.v. or s.c.) whereas anti-insulin antibodies increased notably from $8 \%$ before implantation to $95 \%$ (Biomérieux, Lyon France). The patient had a transient pruritic cutaneous rash, particularly at the injection sites, and specific anti-insulin IgE was detected by a semi-quantitative method. No insulin receptor antibodies were found. Methylprednisolone $(0.5 \mathrm{mg} / \mathrm{kg} /$ day for 10 days, then $2 \mathrm{mg} / \mathrm{kg} /$ day for 2 days), bovine insulin (30 IU/day, associated with regular insulin) and metformin $(2550 \mathrm{mg} /$ day $)$ were ineffective. Methylprednisolone had to be stopped because of the worsening of hyperglycaemia and ketosis. Repeated plasmapheresis (12 times in 1 month), associated with cyclophosphamide $(100 \mathrm{mg} /$ day $)$, resulted in a decrease in anti-insulin antibodies to levels between 35 and $60 \%$. Insulin needs remained, however, above 200 IU/day. Finally, the patient received i.v. immunoglobulins (IVIG), $0.4 \mathrm{mg} / \mathrm{kg} /$ day for 5 days monthly during 5 months. The three first sequences of treatment were followed by a $1 \mathrm{~g}$ i.v. cyclophosphamide bolus. A sustained decrease in anti-insulin antibodies $(<30 \%)$ and insulin doses ( $<50 \mathrm{IU} /$ day) was achieved at the fourth sequence of IVIG (Fig.1), and specific anti-insulin IgE disappeared. During the following 9 months, the patient could be treated by continuous IP insulin infusion, with a mean insulin daily dose of 38 IU/day. Anti-insulin antibodies averaged $34 \%$ and did not require a new IVIG sequence. Because of the recurrence of a third catheter encapsulation, however, the patient was then switched to continuous subcutaneous insulin infusion for the following 4.5 years. During this period, the mean insulin antibodies and the mean insulin doses remained stable at the same levels (26\% and 37 IU/day respectively) without any additional immunomodulating treatment.

The efficacy of corticosteroid therapy in acquired immune insulin resistance has been reported [2]. Plasmapheresis, cyclophosphamide and cyclosporin A proved efficient in type B insulin resistance syndromes [3]. In our patient, corticosteroids were inefficient, and the association plasmapheresis and cyclophosphamide had only a partial and transient effect. The im-

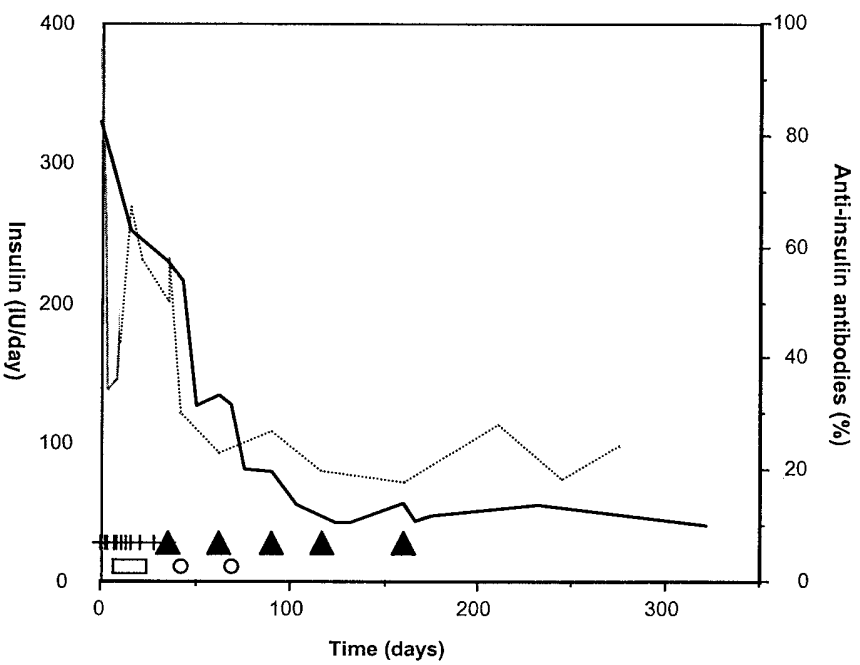

Fig. 1. Changes in anti-insulin antibodies and insulin doses in relation with the treatments of insulin resistance. - Anti-insulin $\mathrm{AB},-$ insulin doses, + plasmapheresis, $\boldsymbol{\Delta}$ immunoglobulins, $\bigcirc$ cyclophosphamide bolus, $\square$ cyclophosphamide continuous treatment

munomodulating effect of IVIG is multifactorial, including blockade of Fc receptors and regulation of the anti-idiotype network. IVIG treatment is effective in several immune diseases and carries a lower risk than immunosuppression [4]. To our knowledge, we report the first treatment of acquired insulin resistance with IVIG. In this case, it was the only therapeutic approach that provided rapid and prolonged efficacy. Therefore, severe acquired immune insulin resistance seems to be a possible indication of IVIG therapy.

Yours sincerely,

H. Hanaire-Broutin, V. Melki, J.P. Tauber

\section{References}

1. Jeandidier N, Boivin S, Sapin R et al. (1995) Immunogenicity of intraperitoneal insulin infusion using programmable implantable devices. Diabetologia 38: 577-584

2. Van Haeften T (1989) Clinical significance of insulin antibodies in insulin treated diabetic patients. Diabetes Care 12: 641-648

3. Eriksson JW, Bremell T, Eliasson B, Fowelin J, Fredriksson L, Yu ZW (1998)Successful treatment with plasmapheresis, cyclophosphamide, and cyclosporin A in type B syndrome of insulin resistance. Diabetes Care 21: 1217-1220

4. Dwyer JM (1992) Manipulating the immune system with immune globulin. N Engl J Med 326: 107-116
Corresponding author: H. Hanaire-Broutin, Department of Diabetology, CHU Rangueil, 1 avenue J. Poulhes, F-31403 Toulouse cedex, France 